\title{
Electrostatic Free Energy and its Variations in Implicit Solvent Models
}

\author{
Jianwei Che, Joachim Dzubiella ${ }^{\dagger}$ Bo Li $;$ and J. Andrew McCammon ${ }^{\S}$
}

December 12, 2007

\begin{abstract}
A mean-field approach to the electrostatics for solutes in electrolyte solution is revisited and rigorously justified. In this approach, an electrostatic free energy functional is constructed that depends solely on the local ionic concentrations. The unique set of such concentrations that minimize this free energy are given by the usual Boltzmann distributions through the electrostatic potential which is determined by the Poisson-Boltzmann equation. This approach is then applied to the variational implicit solvent description of the solvation of molecules [Dzubiella, Swanson, and McCammon, Phys. Rev. Lett., 96, 087802, 2006, and J. Chem. Phys., 124, 084905, 2006]. Care is taken for the singularities of the potential generated by the solute point charges. The variation of the electrostatic free energy with respect to the location change of solute-solvent interfaces, i.e., dielectric boundaries, is derived. Such a variation gives rise to the normal component of the effective surface force per unit surface area that is shown to be attractive to the fixed point charges in the solutes. Two examples of applications are given to validate the analytical results. The first one is a one-dimensional model system resembling, e.g., a charged solute or cavity in a one-dimensional channel. The second one, which is of its own interest, is the electrostatic free energy of a charged sphercal solute immersed in an ionic solution. An analytical formula is derived for the Debye-Hückel approximation of the free energy, extending the classical Born's formula to one that includes ionic
\end{abstract}

*The Genomics Institute of the Novartis Research Foundation, 10675 John Jay Hopkins Drive, San Diego, CA 92121, USA. Email: jche@gnf.org.

†Physik Department (T37), Technische Universität München (TUM), James-Franck-Str., 85748 Garching, Germany. Email: jdzubiel@ph.tum.de.

${ }_{\ddagger}^{\ddagger}$ Department of Mathematics and NSF Center for Theoretical Biological Physics (CTBP), University of California, San Diego, 9500 Gilman Drive, Mail code: 0112, La Jolla, California 92093-0112, USA. Email: bli@math.ucsd.edu.

$\S$ Department of Chemistry and Biochemistry, Department of Pharmacology, and NSF Center for Theoretical Biological Physics (CTBP), University of California, San Diego, La Jolla, California 920930365, USA. Email: jmccammon@ucsd.edu. 
concentrations. Variations of the nonlinear Poisson-Boltzmann free energy are also obtained.

PACS: 82.45.Tv, 82.60.Lf, 87.10.Ed, 87.15.ad, 87.15.B-.

Key words and phrases: ionic solutions, electrostatic free energy, the PoissonBoltzmann equation, implicit solvent, solute-solvent interface, dielectric boundary, free energy variations, the Born-Debye-Hückel formula.

\section{Introduction}

The electrostatic interaction between charged particles gives rise to one of the strongest intermolecular forces that determine the structure and dynamics of an underlying molecular system $[1,2]$. In a mean-field approximation with linear response of the medium, the effective electrostatic free energy of such a system that occupies a region $\Omega$ in $\mathbb{R}^{3}$ can be expressed as a functional of the electrostatic potential $\psi$, charge density $\rho$, and local ionic concentrations $c_{1}, \ldots, c_{M}$. This functional is often given by

$$
\begin{aligned}
& G\left[c_{1}, \ldots, c_{M} ; \psi\right] \\
& \quad=\int_{\Omega}\left\{-\frac{\varepsilon(x)}{8 \pi}|\nabla \psi|^{2}+\rho \psi+\beta^{-1} \sum_{j=1}^{M} c_{j}^{\infty}+\beta^{-1} \sum_{j=1}^{M} c_{j}\left[\ln \left(\Lambda^{3} c_{j}\right)-1\right]-\sum_{j=1}^{M} \mu_{j} c_{j}\right\} d x
\end{aligned}
$$

where $\varepsilon(x)$ is a position-dependent dielectric coefficient, $\beta$ the inverse thermal energy, $c_{j}^{\infty}$ the constant bulk concentration of the $j$ th ionic species that corresponds to the vanishing electrostatic potential, $\Lambda$ the thermal de Broglie wavelength, and $\mu_{j}$ the chemical potential of the $j$ th ionic species. Electrostatics CGS units are used throughout. Extremizing this functional with respect to the concentrations leads to the Boltzmann distribution of concentrations through the potential. Extremizing the functional with respect to the potential leads to the Poisson (PB) equation for the potential; see [3-9]. It has been noticed, however, that this free energy functional is concave with respect to the electrostatic potential. Therefore, the equilibrium concentrations and potential do not minimize this free energy functional; rather they form a saddle point $[3,6,7,9,10]$.

Kralj-Iglic and A. Iglic [11] and Fogolari and Briggs [6] reformulated the electrostatic free energy as a functional depending solely on the local ionic concentrations. In this modified formulation, the non fixed part of the charge density is a linear combination of local ionic concentrations, and the electrostatic potential is uniquely determined by the total charge density through the Poisson equation and related boundary conditions. Minimizing this free energy functional leads to the Boltzmann distributions, which together with the Poisson equation lead to the PB equation for the equilibrium potential. 
Consider now the solvation of molecules. In an implicit or continuum solvent model of such a system, the solvent (such as salted water) molecules are coarse-grained, while the molecule (or solute) atoms are still treated individually. When a sharp solute-solvent interface is assumed, a mean-field approximation of the total free energy of the solvation system is often given by the sum of the nonpolar solute-solvent interaction energy and the polar (electrostatic) interaction energy $[12,13]$. Both of these parts are determined by the location of the solute-solvent interface which is usually taken to be the dielectric boundary.

In most of the current implicit solvent models, the solute-solvent interface is identified as a solvent accessible surface obtained from rolling a probing solvent molecular sphere over the van der Waals surface of solute atoms [14]. The surface energy is typically taken to be proportional to the solvent accessible surface area $[12,13]$. The electrostatic part of the free energy is determined by the PB equation $[4,15,16]$ or the generalized Born (GB) method $[17,18]$ with the dielectric coefficient changing across the dielectric boundary.

In order to couple the polar and non-polar parts of the free energy and to include the curvature effect in a unified treatment, Dzubiella, Swanson, and McCammon $[19,20]$ have recently developed a class of variational implicit solvent models. The basic idea of this approach is to introduce a free energy functional that depends solely on a possible solutesolvent interface. This free energy couples both the non-polar and polar contributions of the system. Minimizing the functional determines the equilibrium solute-solvent interface and the minimum free energy of the solvation system. This interface is an output of the theory. It results automatically from balancing the different contributions of the free energy.

In this work, we first revisit the approach of Kralj-Iglic and Iglic [11] and Fogolari and Briggs [6] to the description of electrostatic free energy for multiple ionic species, and give a rigorous justification of such an approach.

We then apply this approach to the variational implicit solvent modeling of the solvation of molecules, and formulate the corresponding electrostatic free energy that depends only on the solute-solvent interfaces which are taken to be the dielectric boundaries. We demonstrate that this free energy functional is minimized at a unique set of concentrations given by the Boltzmann distributions through the equilibrium electrostatic potential and that this potential satisfies the PB equation.

We further calculate the variation of such free energy with respect to the location change of the dielectric boundary $\Gamma$ locally at a given point $x \in \Gamma$. Our main result is that such variation is given by

$$
\delta_{\Gamma} G[\Gamma](x)=\frac{1}{8 \pi}\left(\frac{1}{\varepsilon_{m}}-\frac{1}{\varepsilon_{s}}\right)\left|\varepsilon_{\Gamma}(x) \nabla \psi_{\Gamma}(x)\right|^{2}+\sum_{j=1}^{M} \beta^{-1} c_{j}^{\infty}\left[e^{-\beta q_{j} \psi_{\Gamma}(x)}-1\right] \quad \forall x \in \Gamma,
$$

where $\Gamma$ is the dielectric boundary, $G[\Gamma]$ is the corresponding minimum electrostatic free energy, and $\delta_{\Gamma}$ denotes the variation with respect to the local change of $\Gamma$ near a given 
point $x$ on $\Gamma$. The parameters in (1.2) are the dielectric coefficient $\varepsilon_{\Gamma}$ which takes one value $\varepsilon_{m}$ in solutes and another $\varepsilon_{s}$ in the solvent, the charge $q_{j}=e z_{j}$ with $e$ the elementary charge and $z_{j}$ the valence of $j$ th ionic species, and the electrostatic potential $\psi_{\Gamma}$ that solves the PB equation; see Section 3 for more details.

The free energy variation (1.2) is calculated with respect to the perturbation along the unit normal to the solute-solvent interface $\Gamma$, pointing from the solute region $\Omega_{m}$ to the solvent region $\Omega_{s}$. Such a variation is a distribution on the dielectric boundary, and can be regarded as the normal component of an effective surface force per unit surface area. We show rigorously that this force is attractive to the fixed solute charges, cf. (3.15).

We finally provide two examples to validate our calculations. The first example is a one-dimensional model problem for which explicit calculations can be carried out to obtain the variation of free energy. This example might have some relevance for the modeling of solutes or cavities (e.g. nanobubbles) confined to one-dimensional channels or nanotubes in electrolyte solutions $[21,22]$. The calculations for this model system show that our main results are correct in form and do not depend on dimensionality. The second example is of its own interest: it is the electrostatic free energy of a spherical solute, with a point charge at its center, immersed in an ionic solution. We derive an explicit formula of the Debye-Hückel (DH) approximation of the potential, which is the solution to the linearized PB equation, and the related approximation of the free energy, cf. (4.6) and (4.8). In general, there is no explicit formula for the potential that is determined by the nonlinear PB equation. We can, however, still obtain the variation of the free energy with respect to the change of the spherical boundary, cf. (4.11).

The rest of this paper is organized as follows: In Section 2, we recall a commonly used description of the electrostatic free energy and point out the fact that the equilibrium concentrations and potential do not minimize such a free energy. We also review the modified approach of Kralj-Iglic and Iglic [11] and Fogolari-Briggs [6] to constructing a free energy that is minimized at equilibrium concentrations which in turn define the equilibrium potential. In Section 3, we apply the modified approach to the implicit solvent modeling of the solvation of molecules, calculate the variation of electrostatic free energy with respect to the location change of the solute-solvent interface, and show that the resulting effective surface force is always attractive to the solutes. The two examples are given in Section 4 to validate our calculations, with some of the tedious calculations given in Appendixes A-C. Finally, in Section 5, we draw conclusions.

\section{Electrostatic free energy of ionic solutions}

\subsection{A commonly used approach}

Consider an ionic solution with $M$ ionic species occupying a region $\Omega$ in $\mathbb{R}^{3}$. One commonly used formulation of the Gibbs electrostatic free energy of the system is given by (1.1) in 
which the charge density is defined by

$$
\rho(x)=\rho_{f}(x)+\sum_{j=1}^{M} q_{j} c_{j}(x)
$$

where $\rho_{f}=\rho_{f}(x)$ represents a known and fixed part of the charge density such as that of point charges in a solute. In Eq. (1.1), the first two terms are the internal electrostatic energy and the third term is the osmotic pressure from the mobile ions. The sum of these three terms constitutes the system enthalpy. The fourth term represents the ideal gas entropy of the free energy. The last term accounts for a constant chemical potential in the system which is treated in the grand canonical ensemble.

Fix $j$ with $1 \leq j \leq M$. Setting the first variation of the free energy $G$ with respect to the concentration $c_{j}$ to zero leads to the Boltzmann distribution for the equilibrium concentrations

$$
c_{j}(x)=c_{j}^{\infty} e^{-\beta q_{j} \psi},
$$

where

$$
c_{j}^{\infty}=\Lambda^{-3} e^{\beta \mu_{j}}
$$

is the constant bulk concentration of the $j$ th ionic species. Setting the first variation of the free energy $G$ with respect to the potential $\psi$ to zero leads to the Poisson equation for the equilibrium potential $\psi$

$$
\nabla \cdot \varepsilon(x) \nabla \psi=-4 \pi \rho(x)
$$

The combination of (2.4), (2.2), and (2.1) leads to the PB equation:

$$
\nabla \cdot \varepsilon(x) \nabla \psi+4 \pi \sum_{j=1}^{M} q_{j} c_{j}^{\infty} e^{-\beta q_{j} \psi}=-4 \pi \rho_{f}(x) .
$$

From (1.1) and (2.1)-(2.3), we obtain the following free energy at the equilibrium potential $\psi$ and equilibrium concentrations $c_{1}, \ldots, c_{M}$ :

$$
G[\psi]=\int_{\Omega}\left[-\frac{\varepsilon(x)}{8 \pi}|\nabla \psi|^{2}+\rho_{f}(x) \psi-\beta^{-1} \sum_{j=1}^{M} c_{j}^{\infty}\left(e^{-\beta q_{j} \psi}-1\right)\right] d x
$$

Notice that this expression defines a concave functional for all possible potentials $\psi$. It is unbounded below, and does not have a minimum value. Rather, It has a unique equilibrium at which the functional is maximized.

We can look more carefully into this issue of energy maximization instead of minimization. For simplicity, let us neglect the concentrations for a moment. The classical electrostatic energy is then the integral of $\rho_{f} \psi / 2$, where $\rho_{f}$ is a fixed charge density and $\psi$ is the potential determined by the Poisson equation (2.4) with $\rho$ replaced by $\rho_{f}$. Here, 
the Poisson equation is not a result of minimizing the electrostatic energy with respect to all possible potentials. In fact, the integral of $\rho \psi / 2$ can be any value in $(-\infty, \infty)$ when $\psi$ is allowed to be any possible function. This means the classical electrostatic energy is neither minimized nor maximized with respect to all possible potentials.

Is there a new form of the electrostatic energy functional whose minimizers satisfy the Poisson equation? We do not know the answer in general. But we can show that such a functional, if exists, can not be of the form

$$
E_{a, b}[\psi]=\int_{\Omega}\left[a \varepsilon(x)|\nabla \psi|^{2}+b \rho_{f} \psi\right] d x
$$

for any constants $a$ and $b$ with $a \neq 0$. In fact, $a>0$ for otherwise $E_{a, b}$ is not minimized. If $a>0$, then $E_{a, b}$ is convex and it has a unique minimizer $\psi$ that satisfies

$$
-2 a \nabla \cdot \varepsilon(x) \nabla \psi+b \rho_{f}=0 .
$$

Comparing with the Poisson equation, we obtain $b<0$. The minimum energy is $E_{a, b}[\psi]$ at this minimizer $\psi$. Thus,

$$
\int_{\Omega} \frac{1}{2} \rho_{f} \psi d x=\int_{\Omega}\left[a \varepsilon(x)|\nabla \psi|^{2}+b \rho_{f} \psi\right] d x=\int_{\Omega}\left[-a \nabla \cdot \varepsilon(x) \nabla \psi+b \rho_{f}\right] \psi d x=\frac{b}{2} \int_{\Omega} \rho_{f} \psi d x .
$$

Since $b<0$, this implies that the energy is 0 , which is in general not true.

\section{$2.2 \quad$ A modified formulation}

Following Kralj-Iglic and Iglic [11] and Fogolari and Briggs [6], and using all the previous notations, we define the electrostatic free energy to be a functional only of the local ionic concentrations $c_{1}, \ldots, c_{M}$ :

$$
\begin{aligned}
G & =G\left[c_{1}, \ldots, c_{M}\right] \\
& =\int_{\Omega}\left\{\frac{1}{2} \rho \psi+\beta^{-1} \sum_{j=1}^{M} c_{j}^{\infty}+\beta^{-1} \sum_{j=1}^{M} c_{j}\left[\ln \left(\Lambda^{3} c_{j}\right)-1\right]-\sum_{j=1}^{M} \int_{\Omega} \mu_{j} c_{j}\right\} d x,
\end{aligned}
$$

where the local charge density $\rho=\rho(x)$ is defined by (2.1) and the local potential $\psi=\psi(x)$ is determined by the Poisson equation (2.4) with certain boundary conditions. Notice that the first part of the integral is the classical representation of the internal electrostatic energy.

We define the partial differential operator

$$
L=-\frac{1}{4 \pi} \nabla \cdot \varepsilon(x) \nabla,
$$


and consider, for simplicity of exposition, the homogeneous Dirichlet boundary condition for the electrostatic potential $\psi$

$$
\psi(x)=0 \quad \text { if } x \in \partial \Omega
$$

where $\partial \Omega$ denotes the boundary of $\Omega$. Since $\varepsilon(x)$ is bounded below by some positive constant, the operator $L$ is linear, self-adjoint, and positive. Therefore, its inverse $L^{-1}$ exists, and is also linear, self-adjoint, and positive. The positivity of $L^{-1}$ means that

$$
\int_{\Omega} \rho\left(L^{-1} \rho\right) d x>0 \quad \text { for any nonzero } \rho \text {. }
$$

The local charge density $\rho=\rho(x)$ and the local electrostatic potential $\psi=\psi(x)$ that satisfies the boundary condition (2.9) therefore determine each other uniquely by $\psi=$ $L^{-1} \rho$ and $\rho=L \psi$.

With all these notations and properties of $L^{-1}$, we can rewrite the free energy $G$ as

$$
\begin{aligned}
G=\int_{\Omega} \frac{1}{2} & {\left[\left(\sum_{j=1}^{M} q_{j} c_{j}\right) L^{-1}\left(\sum_{j=1}^{M} q_{j} c_{j}\right)+2 \rho_{f} L^{-1}\left(\sum_{j=1}^{M} q_{j} c_{j}\right)+\rho_{f} L^{-1} \rho_{f}\right] d x } \\
& +\sum_{j=1}^{M} \int_{\Omega}\left\{\beta^{-1} c_{j}^{\infty}+\beta^{-1} c_{j}\left[\ln \left(\Lambda^{3} c_{j}\right)-1\right]-\mu_{j} c_{j}\right\} d x .
\end{aligned}
$$

Since all $\varepsilon, \rho_{f}, \beta, \Lambda, q_{j}, c_{j}^{\infty}$, and $\mu_{j}(1 \leq j \leq M)$ are known and fixed, this free energy functional depends only on the local ionic concentrations $c_{1}, \ldots, c_{M}$.

Fix a set of concentrations $c=\left(c_{1}, \ldots, c_{M}\right)$, the first variation of the free energy $G$ at $c$ can be identified with a vector-valued distribution whose $j$ th component is

$$
(\delta G[c])_{j}=q_{j} \psi+\beta^{-1} \ln \left(\Lambda^{3} c_{j}\right)-\mu_{j}, \quad j=1, \ldots, M,
$$

where $\psi=L^{-1} \rho$ is the local electrostatic potential generated by the local charge density $\rho=\rho(x)$ given by $(2.1)$ for the concentrations $c_{1}, \ldots, c_{M}$. The equilibrium concentrations, determined by $\delta G[c]=0$, are thus given by the Boltzmann distributions (2.2).

The second variation of $G$ at $c$ is a bilinear form:

$$
\delta^{2} G[c](\hat{c}, \hat{d})=\int_{\Omega}\left(\sum_{j, k=1}^{M} q_{j} q_{k} \hat{c}_{j} L^{-1} \hat{d}_{k}+\sum_{j=1}^{M} \frac{\hat{c}_{j} \hat{d}_{j}}{\beta c_{j}}\right) d x
$$

In particular, for a nonzero perturbation $\hat{c}$, we have by $(2.10)$ that

$$
\delta^{2} G[c](\hat{c}, \hat{c})=\int_{\Omega}\left[\left(\sum_{j=1}^{M} q_{j} \hat{c}_{j}\right) L^{-1}\left(\sum_{j=1}^{M} q_{j} \hat{c}_{j}\right)+\sum_{j=1}^{M} \frac{\hat{c}_{j}^{2}}{\beta c_{j}}\right] d x>0
$$


where we used the fact that each $c_{j}(x)>0$ for any $j$ and any $x \in \Omega$, which follows from the Boltzmann distribution (2.2) and (2.3). Therefore, the free energy $G$ is convex, and the set of equilibrium concentrations $c_{1}, \ldots, c_{M}$ is the unique minimizer of the free energy $G$.

Finally, by (2.7), (2.11), and (2.1)-(2.4), the minimum free energy which is the free energy of the unique equilibrium concentrations $c_{1}, \ldots, c_{M}$, is given by

$$
\begin{aligned}
G_{\text {min }}= & \int_{\Omega}-\frac{1}{2} \rho \psi d x+\int_{\Omega} \rho \psi d x+\sum_{j=1}^{M} \int_{\Omega}\left\{\beta^{-1} c_{j}^{\infty}+\beta^{-1} c_{j}\left[\ln \left(\Lambda^{3} c_{j}\right)-1\right]-\mu_{j} c_{j}\right\} d x \\
= & \int_{\Omega} \frac{1}{8 \pi}[\nabla \cdot \varepsilon(x) \nabla \psi] \psi d x+\int_{\Omega}\left[\rho_{f} \psi+\left(\sum_{j=1}^{M} q_{j} c_{j}^{\infty} e^{-\beta q_{j} \psi}\right) \psi\right] d x \\
& +\sum_{j=1}^{M} \int_{\Omega}\left\{\beta^{-1} c_{j}^{\infty}+\beta^{-1} c_{j}^{\infty} e^{-\beta q_{j} \psi}\left[\ln \left(\Lambda^{3} c_{j}^{\infty}\right)-\beta q_{j} \psi-1\right]-\mu_{j} c_{j}^{\infty} e^{-\beta q_{j} \psi}\right\} d x \\
= & \int_{\Omega}\left[-\frac{\varepsilon(x)}{8 \pi}|\nabla \psi|^{2}+\rho_{f}(x) \psi-\beta^{-1} \sum_{j=1}^{M} c_{j}^{\infty}\left(e^{-\beta q_{j} \psi}-1\right)\right] d x .
\end{aligned}
$$

where $\psi$ is the electrostatic potential corresponding to the free energy minimizing concentrations $c_{1}, \ldots, c_{M}$. Notice that (2.12) is the same as (2.6). But (2.12) is the minimum value of the free energy functional (2.7) of all concentrations, while (2.6) is the value of the free energy functional $(1.1)$ at its extreme $\left(c_{1}, \ldots, c_{M} ; \psi\right)$ which does not minimize (1.1).

\section{Variations of electrostatic free energy in implicit solvent models}

\subsection{Electrostatic free energy in implicit solvent model}

We now consider the solvation of molecules in the framework of variational implicit solvent approach $[19,20]$. We denote by $\Omega$ the entire region of an underlying solvation system. It now consists of the region of biomolecules (or solutes) $\Omega_{m}$, the region of solvent $\Omega_{s}$, and the solute-solvent interface (or molecular surface) $\Gamma$ that separates the solute and solvent regions, cf. Figure 1. As usual, we assume that the interface $\Gamma$ is also the dielectric boundary. This means that the dielectric coefficient in the system is a piecewise constant function:

$$
\varepsilon_{\Gamma}(x)= \begin{cases}\varepsilon_{m} & \text { if } x \in \Omega_{m}, \\ \varepsilon_{s} & \text { if } x \in \Omega_{s},\end{cases}
$$

where $\varepsilon_{m}$ and $\varepsilon_{s}$ are the dielectric constants of the solutes (usually taken as that in the vacuum) and the solvent, respectively. We assume there are $N$ atoms in the solute and 
that the $i$ th atom is located at $x_{i} \in \Omega_{m}$ carrying a point charge $Q_{i}$. We also assume that there are $M$ species of ions in the solvent and that the related notations $c_{j}(x), c_{j}^{\infty}, q_{j}$, and $\mu_{j}$ are the same as before.

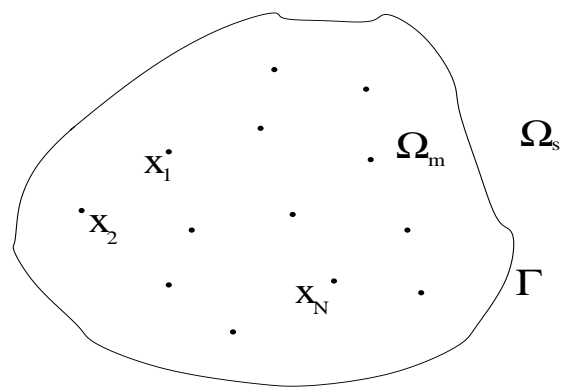

Figure 1. The geometry of a solvation system with implicit solvent.

For a given set of ionic concentrations $c_{1}, \ldots, c_{M}$ of the solvent, the local charge density of the system is now given by

$$
\rho(x)=\rho_{f}(x)+\chi_{s}(x) \sum_{j=1}^{M} q_{j} c_{j}(x),
$$

where $\rho_{f}(x)$ is the known and fixed part of the charge density and $\chi_{s}$ is the characteristic function of the solvent region $\Omega_{s}$ defined by $\chi_{s}(x)=1$ if $x \in \Omega_{s}$ and $\chi_{s}(x)=0$ if $x \notin \Omega_{s}$. In the present case, the fixed part of the charge density is

$$
\rho_{f}(x)=\sum_{i=1}^{N} Q_{i} \delta\left(x-x_{i}\right),
$$

representing all the point charges at the solute atoms, where $\delta\left(x-x_{i}\right)=\delta_{x_{i}}(x)$ is the Dirac delta function concentrated at the $x_{i}$. The presence of the characteristic function $\chi_{s}=\chi_{s}(x)$ in (3.1) means that the ions in the solvent can not penetrate the dielectric boundary $\Gamma$. The electrostatic potential $\psi$ in the entire solvation system is determined by the Poisson equation

$$
\nabla \cdot \varepsilon_{\Gamma}(x) \nabla \psi=-4 \pi \rho(x)
$$

and the boundary condition (2.9).

Similar to (2.7) and (2.11), the electrostatic free energy is now given by

$$
\begin{aligned}
G\left[\Gamma ; c_{1}, \ldots, c_{M}\right]= & \int_{\Omega} \frac{1}{2}\left(\rho_{f}+\chi_{s}(x) \sum_{j=1}^{M} q_{j} c_{j}\right) L^{-1}\left(\rho_{f}+\chi_{s}(x) \sum_{j=1}^{M} q_{j} c_{j}\right) d x \\
& +\sum_{j=1}^{M} \int_{\Omega_{s}}\left\{\beta^{-1} c_{j}^{\infty}+\beta^{-1} c_{j}\left[\ln \left(\Lambda^{3} c_{j}\right)-1\right]-\mu_{j} c_{j}\right\} d x .
\end{aligned}
$$


Notice that some of the integrals are taken over the solvent region $\Omega_{s}$, since the ionic concentrations are zero in the solute region $\Omega_{m}$. Also, the osmotic pressure term is related to the volume of solvent region $\Omega_{s}$ not the entire system region. Notice also that the free energy depends on $\Gamma$ through the characteristic function $\chi_{s}(x)$ and the dielectric coefficient $\varepsilon_{\Gamma}(x)$ in the Poisson equation (3.3).

Repeating the calculations in the previous section and keeping in mind that all the concentrations vanish in the solute region $\Omega_{m}$, we find that the free energy functional is minimized by a unique set of local ionic concentrations $c_{1}, \ldots, c_{M}$ in the solvent region $\Omega_{s}$. They are given by the Boltzmann distributions (cf. (2.2))

$$
c_{j}(x)=\chi_{s}(x) c_{j}^{\infty} e^{-\beta q_{j} \psi(x)},
$$

where $c_{j}^{\infty}(1 \leq j \leq M)$ are given by (2.3). By (3.1)-(3.3) and (3.5), the equilibrium potential $\psi$ is the unique solution of the $\mathrm{PB}$ equation

$$
\nabla \cdot \varepsilon_{\Gamma}(x) \nabla \psi+4 \pi \chi_{s}(x) \sum_{j=1}^{M} q_{j} c_{j}^{\infty} e^{-\beta q_{j} \psi}=-4 \pi \rho_{f}(x) \quad \text { in } \Omega
$$

together with the boundary condition (2.9). Similar to (2.12), the minimum free energy, which is the free energy of the equilibrium system with equilibrium potential and concentrations, is given by

$$
\begin{aligned}
G[\Gamma] & =\min _{c_{1}, \ldots, c_{M}} G\left[\Gamma ; c_{1}, \ldots, c_{M}\right] \\
& =\int_{\Omega}\left[-\frac{\varepsilon_{\Gamma}(x)}{8 \pi}\left|\nabla \psi_{\Gamma}\right|^{2}+\rho_{f}(x) \psi_{\Gamma}-\beta^{-1} \chi_{s}(x) \sum_{j=1}^{M} c_{j}^{\infty}\left(e^{-\beta q_{j} \psi_{\Gamma}}-1\right)\right] d x
\end{aligned}
$$

where $\psi_{\Gamma}$ is the same as $\psi$ that is the solution of the PB equation (3.6). We write $\psi_{\Gamma}$ to emphasize its dependence on $\Gamma$.

Since the fixed charge density $\rho_{f}$ consists of point charges centered at the solute particles $x_{i}(1 \leq i \leq N)$, the potential $\psi_{\Gamma}$ determined by the PB equation (3.6) has singularities at these points $x_{i}$. Thus, the integral of $\left|\nabla \psi_{\Gamma}\right|^{2}$ is taken over the region $\Omega$ minus a ball centered at $x_{i}$ with an infinitesimally small cut-off radii. We can also approximate the delta functions in the fixed charge density by smooth functions of the Gaussian distribution type so that the related integrals are regular. We will take this view in some of our calculations below.

The issue of potential singularities can in fact be resolved rigorously. According to Born's definition [23], the electrostatic free energy is the reversible work needed to charge the system. For a given set of local ionic concentrations $c_{1}, \ldots, c_{M}$, the charge density is given by (3.1) and (3.2), and the corresponding potential is given by $\psi=L^{-1} \rho$, where $L$ is defined by $(2.8)$ (with $\varepsilon(x)$ replaced by $\varepsilon_{\Gamma}(x)$ ) and the boundary condition (2.9). Thus, 
the energy due to the point charges at solute atoms is

$$
\frac{1}{2} \sum_{i=1}^{M} Q_{i}\left(\psi-\psi_{v a c}\right)\left(x_{i}\right)=\frac{1}{2} \sum_{i=1}^{M} Q_{i}\left[L^{-1}\left(\sum_{k=1}^{N} Q_{k} \delta_{x_{k}}\right)-\psi_{v a c}+L^{-1}\left(\chi_{s} \sum_{j=1}^{M} q_{j} c_{j}\right)\right]\left(x_{i}\right),
$$

where

$$
\psi_{v a c}(x)=\sum_{i=1}^{N} \frac{Q_{i}}{\varepsilon_{m}\left|x-x_{i}\right|}
$$

is the reference potential generated by the point charges $Q_{i}$ at $x_{i}$ for all $i=1, \ldots, N$ with the dielectric constant $\varepsilon_{m}$. The energy due to the charge density $\sum_{j=1}^{M} q_{j} c_{j}(x)$ is

$$
\frac{1}{2} \sum_{j=1}^{M} \int_{\Omega_{s}} q_{j} c_{j}(x) \psi(x) d x
$$

where the integral is over $\Omega_{s}$, since we assume the mobile ions can not penetrate the dielectric boundary. The other part of the free energy is contributed by the ionic concentrations in the solvent that do not exist in the reference state. Thus, the total electrostatic free energy is

$$
\begin{aligned}
G\left[\Gamma ; c_{1}, \ldots, c_{M}\right]= & \frac{1}{2} \sum_{i=1}^{M} Q_{i}\left[L^{-1}\left(\sum_{k=1}^{N} Q_{k} \delta_{x_{k}}\right)-\psi_{v a c}+L^{-1}\left(\chi_{s} \sum_{j=1}^{M} q_{j} c_{j}\right)\right]\left(x_{i}\right) \\
& +\sum_{j=1}^{M} \int_{\Omega_{s}}\left\{\frac{1}{2} q_{j} c_{j} \psi+\beta^{-1} c_{j}^{\infty}+\beta^{-1} c_{j}\left[\ln \left(\Lambda^{3} c_{j}\right)-1\right]-\mu_{j} c_{j}\right\} d x .
\end{aligned}
$$

Notice that by Green's formula

$$
L^{-1}\left(\chi_{s} c_{j}\right)\left(x_{k}\right)=\int_{\Omega_{s}} c_{j}\left(L^{-1} \delta_{x_{k}}\right) d x
$$

Thus, the variation of $L^{-1}\left(\chi_{s} c_{j}\right)\left(x_{k}\right)$ with respect to $c_{j}$ is $L^{-1} \delta_{x_{k}}$. Repeating previous calculations, we can thus obtain the Boltzmann distributions (3.5) for the equilibrium concentrations and the $\mathrm{PB}$ equation (3.6) for the equilibrium potential now denoted by $\psi_{\Gamma}$. These, together with the previous two equations for the free energy, lead to the corresponding minimum electrostatic free energy that depends only on the solute-solvent interface $\Gamma$

$$
G[\Gamma]=\frac{1}{2} \sum_{i=1}^{N} Q_{i}\left(\psi_{\Gamma}-\psi_{v a c}\right)\left(x_{i}\right)-\sum_{j=1}^{M} \int_{\Omega_{s}}\left[\frac{1}{2} q_{j} c_{j}^{\infty} \psi_{\Gamma} e^{-\beta q_{j} \psi_{\Gamma}}+\beta^{-1} c_{j}^{\infty}\left(e^{-\beta q_{j} \psi_{\Gamma}}-1\right)\right] d x
$$


Multiplying both sides of Eq. (3.6) by $\chi_{s} \psi=\chi_{s} \psi_{\Gamma}$, applying integration by parts, and using the continuity of both $\psi_{\Gamma}$ and $\varepsilon_{\Gamma} \nabla \psi_{\Gamma}$ across $\Gamma$, we have

$$
\begin{aligned}
\sum_{j=1}^{M} \int_{\Omega_{s}} q_{j} c_{j}^{\infty} \psi_{\Gamma} e^{-\beta q_{j} \psi_{\Gamma}} d x & =-\int_{\Omega_{s}} \frac{\varepsilon_{s}}{4 \pi} \psi_{\Gamma} \nabla^{2} \psi_{\Gamma} d x \\
& =\int_{\Omega_{s}} \frac{\varepsilon_{s}}{4 \pi}\left|\nabla \psi_{\Gamma}\right|^{2} d x+\int_{\Gamma} \frac{1}{4 \pi} \psi_{\Gamma}\left(\varepsilon_{\Gamma} \frac{\partial \psi_{\Gamma}}{\partial n}\right) d S
\end{aligned}
$$

where $n$ denotes the unit normal at $\Gamma$ pointing from $\Omega_{m}$ to $\Omega_{s}$. This and (3.8) lead to another form of the minimum free energy

$$
\begin{aligned}
G[\Gamma]=\frac{1}{2} & \sum_{i=1}^{N} Q_{i}\left(\psi_{\Gamma}-\psi_{v a c}\right)\left(x_{i}\right)-\int_{\Omega_{s}} \frac{\varepsilon_{s}}{8 \pi}\left|\nabla \psi_{\Gamma}\right|^{2} d x-\int_{\Gamma} \frac{1}{8 \pi} \psi_{\Gamma}\left(\varepsilon_{\Gamma} \frac{\partial \psi_{\Gamma}}{\partial n}\right) d S \\
& -\sum_{j=1}^{M} \int_{\Omega_{s}} \beta^{-1} c_{j}^{\infty}\left(e^{-\beta q_{j} \psi_{\Gamma}}-1\right) d x .
\end{aligned}
$$

If we approximate the fixed point charges in (3.2) by a Gaussian type smooth function that vanishes on $\Omega_{s}$, still denoted by $\rho_{f}$, then the two formulas (3.7) and (3.10) only differ by the $\psi_{v a c}$ part which is a constant with respect to the interface $\Gamma$. This follows from that the point charge part in (3.10) is just $(1 / 2) \int_{\Omega} \rho_{f} \psi_{\Gamma} d x$ minus the $\psi_{v a c}$ part and that

$$
\begin{aligned}
\int_{\Gamma} \frac{1}{4 \pi} \psi_{\Gamma}\left(\varepsilon_{\Gamma} \frac{\partial \psi_{\Gamma}}{\partial n}\right) d S & =\int_{\Omega_{m}} \frac{\varepsilon_{m}}{4 \pi}\left|\nabla \psi_{\Gamma}\right|^{2} d x+\int_{\Omega_{m}} \frac{\varepsilon_{m}}{4 \pi}\left(\nabla^{2} \psi_{\Gamma}\right) \psi_{\Gamma} d x \\
& =\int_{\Omega_{m}} \frac{\varepsilon_{m}}{4 \pi}\left|\nabla \psi_{\Gamma}\right|^{2} d x-\int_{\Omega} \rho_{f} \psi_{\Gamma} d x
\end{aligned}
$$

\subsection{Free energy variations with respect to the location change of dielectric boundary}

Fix an arbitrary point $z \in \Gamma$. We define the unit normal to $\Gamma$ at $z$ to be the one pointing from $\Omega_{m}$ to $\Omega_{s}$. Consider a perturbation of the boundary near $z$. Let $\Delta V$ be the volume of the difference between the perturbed and unperturbed boundaries. We assign such a volume a positive sign, if the perturbed boundary near $z$ is on the positive side of the normal direction of $z$ with respect to the unperturbed boundary, and a negative sign otherwise. Let $\Delta G[\Gamma, z]$ be the free energy of the perturbed boundary minus that of the unperturbed one. We define the variation of the free energy $G[\Gamma]$ at $z \in \Gamma$ with respect to the location change of $\Gamma$ near $z \in \Gamma$ in the normal direction to $\Gamma$ to be the limit $\lim _{\Delta V \rightarrow 0} \Delta G[\Gamma, z] / \Delta V$, and denote it by $\delta_{\Gamma} G[\Gamma](z)=\delta_{\Gamma, z} G[\Gamma]$.

Given a function $u_{\Gamma}=u_{\Gamma}(x)$ on $\Omega$ that depends on $\Gamma$. We define the variation of $u_{\Gamma}$ with respect to the local change of boundary $\Gamma$ near a point $z \in \Gamma$ in the normal direction 
at $z$ to be a distribution and denote it by $\delta_{\Gamma, z} u_{\Gamma}$. For a perturbation of $\Gamma$ locally near $z$, we denote by $\Delta u_{\Gamma, z}$ the difference of $u_{\Gamma}$ and its perturbed one. Then, the variation $\delta_{\Gamma, z} u_{\Gamma}$ is defined by

$$
\int_{\Omega} \delta_{\Gamma, z} u_{\Gamma}(x) v(x) d x=\lim _{\Delta V \rightarrow 0} \frac{1}{\Delta V} \int_{\Omega} \Delta u_{\Gamma, z}(x) v(x) d x
$$

for any continuous function $v$ on $\Omega$.

Fix $z \in \Gamma$. For convenience, we shall denote by $\delta_{\Gamma}$ the variation with respect to the local change of $\Gamma$ near $z$. Let $\psi_{\Gamma}=\psi$ be the solution of the PB equation (3.6). By (3.7), we have

$$
\begin{aligned}
\delta_{\Gamma} G[\Gamma]= & \int_{\Omega}\left[\frac{1}{8 \pi} \delta_{\Gamma}\left(\frac{1}{\varepsilon_{\Gamma}(x)}\right)\left|\varepsilon_{\Gamma}(x) \nabla \psi\right|^{2}-\frac{\varepsilon_{\Gamma}(x)}{4 \pi} \nabla \psi \cdot \nabla \delta_{\Gamma} \psi+\rho_{f}(x) \delta_{\Gamma} \psi\right] d x \\
& -\int_{\Omega}\left(\delta_{\Gamma} \chi_{s}(x)\right) \sum_{j=1}^{M} \beta^{-1} c_{j}^{\infty}\left(e^{-\beta q_{j} \psi}-1\right) d x+\int_{\Omega} \chi_{s}(x) \sum_{j=1}^{M} q_{j} c_{j}^{\infty} e^{-\beta q_{j} \psi} \delta_{\Gamma} \psi d x \\
= & \int_{\Omega} \frac{1}{8 \pi} \delta_{\Gamma}\left(\frac{1}{\varepsilon_{\Gamma}(x)}\right)\left|\varepsilon_{\Gamma}(x) \nabla \psi\right|^{2} d x-\int_{\Omega}\left(\delta_{\Gamma} \chi_{s}(x)\right) \sum_{j=1}^{M} \beta^{-1} c_{j}^{\infty}\left(e^{-\beta q_{j}^{\infty} \psi}-1\right) d x
\end{aligned}
$$

where we used

$$
\int_{\Omega}\left[-\frac{\varepsilon_{\Gamma}(x)}{4 \pi} \nabla \psi \cdot \nabla \delta_{\Gamma} \psi+\chi_{s}(x) \sum_{j=1}^{M} q_{j} c_{j}^{\infty} e^{-\beta q_{j} \psi} \delta_{\Gamma} \psi\right] d x=-\int_{\Omega} \rho_{f}(x) \delta_{\Gamma} \psi d x,
$$

which is obtained by multiplying both sides of the PB equation (3.6) by $(1 / 4 \pi) \delta_{\Gamma} \psi$ and integrating the resulting terms over $\Omega$ with the use of integration by parts.

Since $\psi$ and $\varepsilon_{\Gamma} \nabla \psi$ are continuous across the boundary $\Gamma$, we have by (3.11) and Lemma 3.1 below that

$$
\delta_{\Gamma} G[\Gamma](z)=\frac{1}{8 \pi}\left(\frac{1}{\varepsilon_{m}}-\frac{1}{\varepsilon_{s}}\right)\left|\varepsilon_{\Gamma}(z) \nabla \psi(z)\right|^{2}+\sum_{j=1}^{M} \beta^{-1} c_{j}^{\infty}\left(e^{-\beta q_{j} \psi(z)}-1\right) .
$$

A different argument is as follows. First, the given boundary $\Gamma$ determines the potential $\psi$ that is the solution of the PB equation (3.6). Now perturb $\Gamma$ locally at a point $z \in \Gamma$ with the perturbation of order $\tau$. This leads to the variation of the dielectric coefficient $\varepsilon_{\Gamma}+\tau \delta_{\Gamma} \varepsilon_{\Gamma}$, up to the leading orders. This variation leads to the potential variation $\psi+\tau \delta_{\Gamma} \psi$, up to the leading orders. The corresponding free energy is then given by

$$
G\left[\psi+\tau \delta_{\Gamma} \psi\right]=G[\psi]+\tau \delta_{\Gamma} G+O\left(\tau^{2}\right) \quad \text { as } \tau \rightarrow 0 .
$$

On the other hand, it follows from (3.7) that

$$
G\left[\psi+\tau \delta_{\Gamma} \psi\right]=\int_{\Omega}\left[-\frac{1}{8 \pi}\left(\varepsilon_{\Gamma}+\tau \delta_{\Gamma} \varepsilon_{\Gamma}\right)\left|\nabla\left(\psi+\tau \delta_{\Gamma} \psi\right)\right|^{2}+\rho_{f}\left(\psi+\delta_{\Gamma} \psi\right)\right.
$$




$$
\begin{gathered}
\left.-\left(\chi_{s}+\delta_{\Gamma} \chi_{s}\right) \sum_{j=1}^{M} \beta^{-1} c_{j}^{\infty}\left(e^{-\beta q_{j}\left(\psi+\tau \delta_{\Gamma} \psi\right)}-1\right)\right] d x+O\left(\tau^{2}\right) \\
=G[\psi]+\tau \int_{\Omega}\left[-\frac{1}{4 \pi} \nabla \psi \cdot \delta_{\Gamma} \psi-\frac{1}{8 \pi} \delta_{\Gamma} \varepsilon_{\Gamma}|\nabla \psi|^{2}+\rho_{f} \delta_{\Gamma} \psi\right. \\
\left.\quad+\chi_{s} \sum_{j=1}^{M} q_{j} c_{j}^{\infty} e^{-\beta q_{j} \psi} \delta_{\Gamma} \psi+\delta_{\Gamma} \chi_{s} \sum_{j=1}^{M} \beta^{-1} c_{j}^{\infty}\left(e^{-\beta q_{j} \psi}-1\right)\right] d x+O\left(\tau^{2}\right) \\
=G[\psi]+\tau \int_{\Omega}\left[-\frac{1}{8 \pi} \delta_{\Gamma} \varepsilon_{\Gamma}|\nabla \psi|^{2}+\delta_{\Gamma} \chi_{s} \sum_{j=1}^{M} \beta^{-1} c_{j}^{\infty}\left(e^{-\beta q_{j} \psi}-1\right)\right] d x+O\left(\tau^{2}\right),
\end{gathered}
$$

where we used (3.12). From the above two equations and Lemma 3.1 below, we obtain the same result

$$
\begin{aligned}
\delta_{\Gamma} G[\Gamma](z) & =\int_{\Omega}\left[-\frac{1}{8 \pi} \delta_{\Gamma} \varepsilon_{\Gamma}|\nabla \psi|^{2}+\delta_{\Gamma} \chi_{s} \sum_{j=1}^{M} \beta^{-1} c_{j}^{\infty}\left(e^{-\beta q_{j} \psi}-1\right)\right] d x \\
& =\int_{\Omega} \frac{1}{8 \pi} \delta_{\Gamma}\left(\frac{1}{\varepsilon_{\Gamma}}\right)\left|\varepsilon_{\Gamma} \nabla \psi\right|^{2} d x+\int_{\Omega} \delta_{\Gamma} \chi_{s} \sum_{j=1}^{M} \beta^{-1} c_{j}^{\infty}\left(e^{-\beta q_{j} \psi}-1\right) d x \\
& =\frac{1}{8 \pi}\left(\frac{1}{\varepsilon_{m}}-\frac{1}{\varepsilon_{s}}\right)\left|\varepsilon_{\Gamma}(z) \nabla \psi(z)\right|^{2}+\sum_{j=1}^{M} \beta^{-1} c_{j}^{\infty}\left[e^{-\beta q_{j} \psi(z)}-1\right] .
\end{aligned}
$$

Let $c^{\infty}=\sum_{j=1}^{M} c_{j}^{\infty}>0$. Since the function $f(t)=e^{-\beta \psi t}-1$ for each $\psi=\psi(z)$ is convex, i.e., $f^{\prime \prime}(t)>0$, we have by Jensen's inequality that

$$
\sum_{j=1}^{M} c_{j}^{\infty}\left[e^{-\beta q_{j} \psi(z)}-1\right]=c^{\infty} \sum_{j=1}^{M} \frac{c_{j}^{\infty}}{c^{\infty}}\left[e^{-\beta q_{j} \psi(z)}-1\right] \geq c^{\infty}\left[e^{-\left(\beta \psi(z) / c^{\infty}\right) \sum_{j=1}^{M} c_{j}^{\infty} q_{j}}-1\right]=0,
$$

where we used the electrostatic neutrality $\sum_{j=1}^{M} q_{j} c_{j}^{\infty}=0$. Therefore, since $0<\varepsilon_{m}<\varepsilon_{s}$, the variation $\delta_{\Gamma} G[\Gamma]$ of the electrostatic free energy with respect to the local location change of the dielectric boundary $\Gamma$, along the normal direction from $\Omega_{s}$ to $\Omega_{s}$, is always positive. Notice that $-\delta_{\Gamma} G[\Gamma]$ is the normal component of surface force per unit surface area. Therefore, the resulting force is attractive to the solutes.

We end this section with the lemma used above.

Lemma 3.1 Let $u_{\Gamma}=u_{\Gamma}(x)$ be a piecewise constant function on $\Omega$ with

$$
u(x)= \begin{cases}u_{m} & \text { if } x \in \Omega_{m}, \\ u_{s} & \text { if } x \in \Omega_{s}\end{cases}
$$


where $u_{m}$ and $u_{s}$ are two constants. Let $v=v(x)$ be a function on $\Omega$ that is continuous across the interface $\Gamma$. Then

$$
\int_{\Omega}\left(\delta_{\Gamma, z} u\right) v d x=\left(u_{m}-u_{s}\right) v(z) \quad \forall z \in \Gamma .
$$



Figure 2. Perturbation of the interface. The dotted line represents the perturbed interface location.

Proof of Lemma. The difference between the perturbed new and unperturbed boundaries near a given point $z \in \Gamma$ is the region $\Delta \Omega$ with volume $\Delta V$. Consider first the case that the volume $\Delta V$ is assigned a negative sign as in Figure 2. We have

$$
\begin{aligned}
\int_{\Omega}\left(\delta_{\Gamma, z} u\right) v d x & =\lim _{\Delta V \rightarrow 0} \frac{1}{-\Delta V}\left[\int_{\Delta \Omega} u_{s} v(x) d x-\int_{\Delta \Omega} u_{m} v(x)\right] d x \\
& =\left(u_{m}-u_{s}\right) \lim _{\Delta V \rightarrow 0} \frac{1}{\Delta V} \int_{\Delta \Omega} v(x) d x=\left(u_{m}-u_{s}\right) v(z) .
\end{aligned}
$$

The other case with the volume assigned a positive sign can be treated similarly.

\section{Examples}

\subsection{A one-dimensional model problem}

Consider a model system that occupies a one-dimensional domain $\left(\alpha_{-}, \alpha_{+}\right)$. Let $f=f(x)$ be a given continuous function on $\left[\alpha_{-}, \alpha_{+}\right]$, resembling a fixed charge density in a solution. Let $W$ be a smooth function on $\mathbb{R}$ that satisfies: (1) $W$ is strictly convex; (2) inf $W>-\infty$; and $(3) W( \pm \infty)=+\infty$. For any "interface" $\gamma \in\left(\alpha_{-}, \alpha_{+}\right)$, we define the "free energy"

$$
G[\gamma]=\min _{v \in H_{0}^{1}\left(\alpha_{-}, \alpha_{+}\right)}\left\{\int_{\alpha_{-}}^{\alpha_{+}}\left[\frac{\varepsilon_{\gamma}(x)}{2} v^{\prime}(x)^{2}-f(x) v(x)\right] d x+\int_{\gamma}^{\alpha_{+}} W(v(x)) d x\right\},
$$

where $H_{0}^{1}\left(\alpha_{-}, \alpha_{+}\right)$denotes the space of all functions $v$ such that $v$ and $v^{\prime}$ are squareintegrable on $\left(\alpha_{-}, \alpha_{+}\right)$and $v\left(\alpha_{-}\right)=v\left(\alpha_{+}\right)=0$,

$$
\varepsilon_{\gamma}(x)= \begin{cases}\varepsilon_{-} & \text {if } x<\gamma \\ \varepsilon_{+} & \text {if } x>\gamma\end{cases}
$$


and $\varepsilon_{-}$and $\varepsilon_{+}$are two positive constants, like the dielectric constants in the previous sections. Notice that the second integral in (4.1) is taken over $\left(\gamma, \alpha_{+}\right)$. Up to a sign, this term resembles the Boltzmann distribution term in the PB free energy, cf. (3.7).

By a series of calculations that are summarized in Appendix A, we obtain the variation of the energy

$$
G^{\prime}[\gamma]=\frac{1}{2}\left(\frac{1}{\varepsilon_{+}}-\frac{1}{\varepsilon_{-}}\right)\left[\varepsilon_{\gamma} u_{\gamma}^{\prime}(\gamma)\right]^{2}-W\left(u_{\gamma}(\gamma)\right) \quad \forall \gamma \in\left(\alpha_{-}, \alpha_{+}\right)
$$

Notice that the functional $G[\gamma]$ defined in (4.1) differs from $G[\Gamma]$ in (3.7), among many others, by a sign. Thus, the result (4.2) agrees with our general formula (1.2) in the form.

\subsection{A charged spherical solute in an ionic solution}

Consider a spherical solute (colloid, macroion, protein, etc.) centered at the origin with radius $R$ in an electrolyte solution. Assume the solute carries a single point charge $Q$ at its center. Assume also there are $M$ ionic species in the solvent with all the related notations $c_{j}(x), c_{j}^{\infty}, \psi, q_{j}$, and $\mu_{j}$ same as before. The solute and solvent regions are

$$
\Omega_{-}(R)=\left\{x \in \mathbb{R}^{3}:|x|<R\right\} \quad \text { and } \quad \Omega_{+}(R)=\left\{x \in \mathbb{R}^{3}:|x|>R\right\},
$$

respectively, and the solute-solvent interface is

$$
\Gamma=\Gamma_{R}=\left\{x \in \mathbb{R}^{3}:|x|=R\right\}
$$

We write $\varepsilon_{R}=\varepsilon_{\Gamma}, \varepsilon_{-}=\varepsilon_{m}$, and $\varepsilon_{+}=\varepsilon_{s}$. Hence,

$$
\varepsilon_{R}(x)= \begin{cases}\varepsilon_{-} & \text {if }|x|<R \\ \varepsilon_{+} & \text {if }|x|>R\end{cases}
$$

We also denote by $\chi_{R}$ the characteristic function of the solvent region $\Omega_{+}(R)$. To indicate the dependence on $R$, we denote the spherically symmetrical, equilibrium electrostatic potential by $\psi_{R}$ and write $\psi_{R}(x)=\psi_{R}(r)$ for any $x$ with $|x|=r$.

By (3.10), the free energy as a function of the radius $R$ is given by

$$
\begin{aligned}
G[R]=\frac{1}{2} & Q\left(\psi_{R}-\psi_{v a c}\right)(0)-\frac{\varepsilon_{+}}{2} \int_{R}^{\infty}\left|\psi_{R}^{\prime}(r)\right|^{2} r^{2} d r-\frac{\varepsilon_{-} R^{2}}{2} \psi_{R}(R) \psi_{R}^{\prime}(R-) \\
& -4 \pi \beta^{-1} \sum_{j=1}^{M} c_{j}^{\infty} \int_{R}^{\infty}\left[e^{-\beta q_{j} \psi_{R}(r)}-1\right] r^{2} d r
\end{aligned}
$$

The derivative $G^{\prime}[R]$ will be derived below for the potential $\psi_{R}$ determined by either the linearized or the fully nonlinear PB equation. 
If we perturb the spherical boundary of the solute by changing $R$ to $R+\Delta R$, then the volume change is

$$
\Delta V=\frac{4 \pi}{3}(R+\Delta R)^{3}-\frac{4 \pi}{3} R^{3}=4 \pi R^{2} \Delta R+O\left((\Delta R)^{2}\right) .
$$

Consequently, the variation of the free energy with respect to the location change of the boundary $\Gamma_{R}$ is

$$
\delta_{R} G[R]=\lim _{\Delta V \rightarrow 0} \frac{G[R+\Delta R]-G[R]}{\Delta V}=\frac{G^{\prime}[R]}{4 \pi R^{2}} .
$$

\subsubsection{The Debye-Hückel approximation}

The Debye-Hückel (DH) approximation of the electrostatic potential, still denoted by $\psi_{R}$, is the solution to the linearized $\mathrm{PB}$ equation

$$
\nabla \cdot \varepsilon_{R}(x) \nabla \psi_{R}-\varepsilon_{+} \kappa^{2} \chi_{R}(x) \psi_{R}=-4 \pi Q \delta(x) \quad \text { in } \mathbb{R}^{3},
$$

together with some boundary condition which we assume to be $\psi_{R}(\infty)=0$. Here,

$$
\kappa=\sqrt{\frac{4 \pi \beta \sum_{j=1}^{M} q_{j}^{2} c_{j}^{\infty}}{\varepsilon_{+}}}
$$

defines the inverse electrostatic decay length (or ionic strength) of the solution. The DB approximation is valid for weak electrostatic potentials and/or moderate to high salt concentrations with high electrostatic screening.

The linearized PB equation (4.5) with our boundary conditions can be equivalently written as

$$
\left\{\begin{array}{l}
\varepsilon_{-} \nabla^{2} \psi_{R}=-4 \pi Q \delta(x) \quad \text { for }|x|<R \\
\nabla^{2} \psi_{R}-\kappa^{2} \psi=0 \quad \text { for }|x|>R \\
\psi_{R}(R-0)=\psi_{R}(R+0) \\
\varepsilon_{-} \psi_{R}^{\prime}(R-0)=\varepsilon_{+} \psi_{R}^{\prime}(R+0) \\
\psi_{R}(\infty)=0
\end{array}\right.
$$

Solving this system of equations using the spherical symmetry, we get the DH potential

$$
\psi_{R}(r)= \begin{cases}\frac{Q}{R}\left(\frac{1}{\varepsilon_{+}}-\frac{1}{\varepsilon_{-}}\right)-\frac{\kappa Q}{\varepsilon_{+}(1+\kappa R)}+\frac{Q}{\varepsilon_{-} r} & \text { if } r<R, \\ \frac{Q}{\varepsilon_{+}(1+\kappa R) r} e^{-\kappa(r-R)} & \text { if } r>R .\end{cases}
$$

By (4.3) and a series of calculations that are presented in Appendix B, we obtain an approximation of the free energy

$$
G[R]=\frac{Q^{2}}{2 R}\left(\frac{1}{\varepsilon_{+}}-\frac{1}{\varepsilon_{-}}\right)-\frac{\kappa Q^{2}(1+2 \kappa R)}{4 \varepsilon_{+}(1+\kappa R)^{2}}
$$




$$
-4 \pi \beta^{-1} \sum_{j=1}^{M} c_{j}^{\infty} \int_{R}^{\infty}\left[e^{-\left(p_{j} / r\right) e^{-\kappa(r-R)}}-1\right] r^{2} d r
$$

where $p_{j}=\beta q_{j} Q /\left[\varepsilon_{+}(1+\kappa R)\right]$. If we further replace the exponential term by its linear approximation and use the electrostatic neutrality $\sum_{j=1}^{M} q_{j} c_{j}^{\infty}=0$, we obtain

$$
G[R]=\frac{Q^{2}}{2 R}\left(\frac{1}{\varepsilon_{+}}-\frac{1}{\varepsilon_{-}}\right)-\frac{\kappa Q^{2}}{2 \varepsilon_{+}(1+\kappa R)} .
$$

It then follows from (4.4) and (4.8) that the variation of the free energy $G[R]$ with respect to the local change of the spherical boundary is

$$
\delta_{R} G[R]=\frac{Q^{2}}{8 \pi R^{4}}\left(\frac{1}{\varepsilon_{-}}-\frac{1}{\varepsilon_{+}}\right)+\frac{\kappa^{2} Q^{2}}{2 \varepsilon_{+}(1+\kappa R)^{2}},
$$

which is always positive, since in general $0<\varepsilon_{-}<\varepsilon_{+}$.

Notice that if there are no ions, then $\kappa=0$ and thus the second term in $G[R]$ in (4.8) vanishes. The resulting formula is the classical Born's formula. Notice also that an approximation different from (4.8) will be obtained if we use the formula (3.8) instead of (3.10), since the equivalence of these two formulas (3.8) and (3.10) follows from the nonlinear PB equation for the potential. However, different DH approximations only differ by terms linear in the ionic strength $\kappa$. We shall pick up (4.8) and call it the Born-Debye-Hückel (BDH) formula.

\subsubsection{The fully nonlinear Poisson-Boltzmann description}

Consider now the general case that the potential $\psi_{R}$ is the solution to the fully nonlinear PB equation (cf. (3.6))

$$
\nabla \cdot \varepsilon_{\Gamma}(x) \nabla \psi_{R}+4 \pi \chi_{R}(x) \sum_{j=1}^{M} q_{j} c_{j}^{\infty} e^{-\beta q_{j} \psi_{R}}=-4 \pi Q \delta(x) \quad \text { in } \mathbb{R}^{3},
$$

together with the boundary condition $\psi_{R}(\infty)=0$. In this case, we do not have an explicit formula of the equilibrium potential $\psi_{R}$. But such potential exists and is unique. Moreover, the free energy is given by (4.3).

By a series of calculations presented in Appendix C, we obtain the derivative

$$
G^{\prime}[R]=\frac{Q^{2}}{2 R^{2}}\left(\frac{1}{\varepsilon_{-}}-\frac{1}{\varepsilon_{+}}\right)+4 \pi R^{2} \beta^{-1} \sum_{j=1}^{M} c_{j}^{\infty}\left[e^{-\beta q_{j} \psi_{R}(R)}-1\right] .
$$

It then follows from (4.4) that the variation of the free energy with respect to the location change of the boundary $\Gamma_{R}$ is

$$
\delta_{R} G[R]=\frac{Q^{2}}{8 \pi R^{4}}\left(\frac{1}{\varepsilon_{-}}-\frac{1}{\varepsilon_{+}}\right)+\beta^{-1} \sum_{j=1}^{M} c_{j}^{\infty}\left[e^{-\beta q_{j} \psi_{R}(R)}-1\right] .
$$


Notice that $G^{\prime}[R]$ is the directional derivative along the direction of increasing the $R$ value, i.e., in the normal direction pointing from $\Omega_{-}$to $\Omega_{+}$. Notice also that by (C.3) $\left|\varepsilon_{+} \psi_{R}^{\prime}(R+)\right|=\left|\varepsilon_{-} \psi_{R}^{\prime}(R-)\right|=Q / R^{2}$. Therefore, if we apply our formula (1.2) (cf. also (3.13)) with $\varepsilon_{m}=\varepsilon_{-}, \varepsilon_{s}=\varepsilon_{+}$, we get the same result.

\section{Conclusions}

In this work, we revisited the approach of Kralj-Iglic and Iglic [11] and Fogolari and Briggs [6] to the construction of electrostatic free energy of an ionic solution that depends only on the local ionic concentrations. We applied this approach to the variational implicit solvent description of the solvation of molecules. The electrostatic free energy is now defined to be a functional of all possible solute-solvent interfaces that we take as dielectric boundaries.

Our key result is the formula (1.2) of the variation of such free energy with respect to the location change of the dielectric boundary. Such a variation defines the normal component, along the normal pointing from the solute to solvent regions, of an effective surface force per unit surface area. Our analysis shows that this force is attractive to the solute charges. This gives a quantitative interpretation in the frame work of implicit solvent the following well known result: under the combined influence of electric field generated by solute charges and their polarization in the surrounding medium which is electrostatic neutral, an additional potential energy emerges and drives the surrounding molecules to the solutes; see [1].

The validation of our calculations is given through two examples. The first example is a one-dimensional model system which might have some relevance for the solvation in highly confined systems. In the second example which is of particular interest, we extended Born's calculation of the free energy of a spherical solute with a point charge at its center to that for such a charged solute in an ionic solution. Within the DH (linearized PB) approximation, the free energy and its variation are given by the classical Born's free energy and its corresponding variation with corrections that are linear and quadratic, respectively, in the ionic strength in the limit of dilute ionic solution.

In our calculations, we used the zero Dirichlet boundary condition for the electrostatic potential. The general case of non-zero Dirichlet boundary condition is of interest, since the resulting Boltzmann distribution can be different. This is worth of further study.

It is well-known that the approach based on the PB equation neglects steric effects and correlation caused by the coarse structure of the solvent and the short-range repulsion between the ions. Some mean field models have been proposed to include the ionic steric effects $[5,11]$. One of our current studies is to apply our method of analysis to these models. 


\section{Appendix A.}

By standard arguments in the calculus of variations, the minimization in (4.1) is attained by a unique function $u_{\gamma} \in H_{0}^{1}\left(\alpha_{-}, \alpha_{+}\right)$which is also the unique weak solution of the Euler-Lagrange equation

$$
\left\{\begin{array}{l}
-\left(\varepsilon_{\gamma} u_{\gamma}^{\prime}\right)^{\prime}+\chi_{\left(\gamma, \alpha_{+}\right)} W^{\prime}\left(u_{\gamma}\right)=f \quad \text { in }\left(\alpha_{-}, \alpha_{+}\right) \\
u_{\gamma}\left(\alpha_{-}\right)=u_{\gamma}\left(\alpha_{+}\right)=0
\end{array}\right.
$$

where $\chi_{\left(\gamma, \alpha_{+}\right)}$is the characteristic function of the interval $\left(\gamma, \alpha_{+}\right)$, i.e., $\chi_{\left(\gamma, \alpha_{+}\right)}(x)=1$ if $x \in\left(\gamma, \alpha_{+}\right)$and $\chi_{\left(\gamma, \alpha_{+}\right)}(x)=0$ if $x \notin\left(\gamma, \alpha_{+}\right)$. Mathematically, the equation (A.1) is understood in the sense of distributions. This means that $u_{\gamma}$ is characterized by $u_{\gamma} \in$ $H_{0}^{1}\left(\alpha_{-}, \alpha_{+}\right)$and

$\int_{\alpha_{-}}^{\alpha_{+}} \varepsilon_{\gamma}(x) u_{\gamma}^{\prime}(x) v^{\prime}(x) d x+\int_{\gamma}^{\alpha_{+}} W^{\prime}\left(u_{\gamma}(x)\right) v(x) d x=\int_{\alpha_{-}}^{\alpha_{+}} f(x) v(x) d x \quad \forall v \in H_{0}^{1}\left(\alpha_{-}, \alpha_{+}\right)$.

It is easy to verify that $u_{\gamma} \in H_{0}^{1}\left(\alpha_{-}, \alpha_{+}\right)$is also the unique solution of the following interface problem:

$$
\left\{\begin{array}{l}
-\left(\varepsilon_{-} u_{\gamma}^{\prime}\right)^{\prime}=f \quad \text { for } \alpha_{-}<x<\gamma \\
-\left(\varepsilon_{+} u_{\gamma}^{\prime}\right)^{\prime}+W^{\prime}\left(u_{\gamma}\right)=f \quad \text { for } \gamma<x<\alpha_{+} \\
u_{\gamma}(\gamma-0)=u_{\gamma}(\gamma+0) \\
\varepsilon_{-} u_{\gamma}^{\prime}(\gamma-0)=\varepsilon_{+} u_{\gamma}^{\prime}(\gamma+0) \\
u_{\gamma}\left(\alpha_{-}\right)=0 \\
u_{\gamma}\left(\alpha_{+}\right)=0
\end{array}\right.
$$

The third and fourth equations in this system form the interface conditions that describe the continuity of the solution (e.g., an electrostatic potential) and of the "normal flux" $\varepsilon_{\gamma} u_{\gamma}^{\prime}$ (e.g., the electrostatic displacement) across the interface $\gamma$.

Solving this system of equations (A.3), we obtain

$$
u_{\gamma}(x)=\left\{\begin{array}{l}
-\frac{1}{\varepsilon_{-}} \int_{\gamma}^{x} g_{\gamma}(y) d y+c_{-} x+d_{-} \quad \text { if } \alpha_{-}<x<\gamma, \\
\frac{1}{\varepsilon_{+}} \int_{\gamma}^{x}\left[\int_{\gamma}^{y} W^{\prime}\left(u_{\gamma}(z)\right) d z\right] d y-\frac{1}{\varepsilon_{+}} \int_{\gamma}^{x} g_{\gamma}(y) d y+c_{+} x+d_{+} \quad \text { if } \gamma<x<\alpha_{+},
\end{array}\right.
$$

where

$$
g_{\gamma}(x)=\int_{\gamma}^{x} f(y) d y \quad \forall x \in\left(\alpha_{-}, \alpha_{+}\right)
$$


and all $c_{ \pm}=c_{ \pm}(\gamma)$ and $d_{ \pm}=d_{ \pm}(\gamma)$ are constants that depend on $\gamma$. By the interface conditions (the third and forth equations in (A.3)) and the boundary conditions (the fifth and sixth equations in (A.3)), we obtain through a series of calculations

$$
\begin{aligned}
& c_{-}=c_{-}(\gamma)=\frac{\varepsilon_{+}}{\Delta}\left[\frac{1}{\varepsilon_{+}} \int_{\gamma}^{\alpha_{+}}\left[\int_{\gamma}^{y} W^{\prime}\left(u_{\gamma}(z)\right) d z\right] d y-\frac{1}{\varepsilon_{+}} \int_{\gamma}^{\alpha_{+}} g_{\gamma}(x) d x+\frac{1}{\varepsilon_{-}} \int_{\gamma}^{\alpha_{-}} g_{\gamma}(x) d x\right], \\
& c_{+}=c_{+}(\gamma)=\frac{\varepsilon_{-}}{\Delta}\left[\frac{1}{\varepsilon_{+}} \int_{\gamma}^{\alpha_{+}}\left[\int_{\gamma}^{y} W^{\prime}\left(u_{\gamma}(z)\right) d z\right] d y-\frac{1}{\varepsilon_{+}} \int_{\gamma}^{\alpha_{+}} g_{\gamma}(x) d x+\frac{1}{\varepsilon_{-}} \int_{\gamma}^{\alpha_{-}} g_{\gamma}(x) d x\right], \\
& d_{-}=d_{-}(\gamma)=\frac{1}{\varepsilon_{-}} \int_{\gamma}^{\alpha_{-}} g_{\gamma}(x) d x-\alpha_{-} c_{-}(\gamma), \\
& d_{+}=d_{+}(\gamma)=-\frac{1}{\varepsilon_{+}} \int_{\gamma}^{\alpha_{+}}\left[\int_{\gamma}^{y} W^{\prime}\left(u_{\gamma}(z)\right) d z\right] d y+\frac{1}{\varepsilon_{+}} \int_{\gamma}^{\alpha_{+}} g_{\gamma}(x) d x-\alpha_{+} c_{+}(\gamma),
\end{aligned}
$$

where

$$
\Delta=\Delta(\gamma)=\operatorname{det}\left(\begin{array}{cccc}
\gamma & -\gamma & 1 & -1 \\
\varepsilon_{-} & -\varepsilon_{+} & 0 & 0 \\
\alpha_{-} & 0 & 1 & 0 \\
0 & \alpha_{+} & 0 & 1
\end{array}\right)=\varepsilon_{-}\left(\gamma-\alpha_{+}\right)+\varepsilon_{+}\left(\alpha_{-}-\gamma\right)<0
$$

Setting $v=u_{\gamma}$ in (A.2), we get

$$
\int_{\alpha_{-}}^{\alpha_{+}} f(x) u_{\gamma}(x) d x=\int_{\alpha_{-}}^{\alpha_{+}} \varepsilon_{\gamma}(x) u_{\gamma}^{\prime}(x)^{2} d x+\int_{\gamma}^{\alpha_{+}} W^{\prime}\left(u_{\gamma}(x)\right) u_{\gamma}(x) d x .
$$

Thus, the free energy is given by (cf. (4.1))

$$
\begin{aligned}
G[\gamma] & =\int_{\alpha_{-}}^{\alpha_{+}}\left[\frac{\varepsilon_{\gamma}(x)}{2} u_{\gamma}^{\prime}(x)^{2}-f(x) u_{\gamma}(x)\right] d x+\int_{\gamma}^{\alpha_{+}} W\left(u_{\gamma}(x)\right) d x \\
& =-\frac{1}{2} \int_{\alpha_{-}}^{\alpha_{+}} \varepsilon_{\gamma}(x) u_{\gamma}^{\prime}(x)^{2} d x+\int_{\gamma}^{\alpha_{+}}\left[W\left(u_{\gamma}(x)\right)-W^{\prime}\left(u_{\gamma}(x)\right) u_{\gamma}(x)\right] d x \\
& =-\frac{\varepsilon_{-}}{2} \int_{\alpha_{-}}^{\gamma} u_{\gamma}^{\prime}(x)^{2} d x-\frac{\varepsilon_{+}}{2} \int_{\gamma}^{\alpha_{+}} u_{\gamma}^{\prime}(x)^{2} d x+\int_{\gamma}^{\alpha_{+}}\left[W\left(u_{\gamma}(x)\right)-W^{\prime}\left(u_{\gamma}(x)\right) u_{\gamma}(x)\right] d x .
\end{aligned}
$$

From (A.4) and (A.5), we can calculate $u_{\gamma}^{\prime}(x)$ and $(d / d \gamma) u_{\gamma}^{\prime}(x)$. By (A.4) and the continuity of $\varepsilon_{\gamma} u_{\gamma}^{\prime}$ at $\gamma$ (cf. the fourth equation of (A.3)), we have $\varepsilon_{-} c_{-}(\gamma)=\varepsilon_{+} c_{+}(\gamma)$, and hence $\varepsilon_{-} c_{-}^{\prime}(\gamma)=\varepsilon_{+} c_{+}^{\prime}(\gamma)$. Combining all these and the boundary conditions (cf. the last two equations in (A.3)), we obtain the variation of the free energy at $\gamma$

$$
\begin{aligned}
G^{\prime}[\gamma]=- & \frac{1}{2 \varepsilon_{-}}\left[\varepsilon_{-} u_{\gamma}^{\prime}(\gamma-0)\right]^{2}-\varepsilon_{-} \int_{\alpha_{-}}^{\gamma} u_{\gamma}^{\prime}(x) \frac{d}{d \gamma} u_{\gamma}^{\prime}(x) d x \\
& +\frac{1}{2 \varepsilon_{+}}\left[\varepsilon_{+} u_{\gamma}^{\prime}(\gamma+0)\right]^{2}-\varepsilon_{+} \int_{\gamma}^{\alpha_{+}} u_{\gamma}^{\prime}(x) \frac{d}{d \gamma} u_{\gamma}^{\prime}(x) d x
\end{aligned}
$$




$$
\begin{aligned}
& -\left[W\left(u_{\gamma}(\gamma)\right)-W^{\prime}\left(u_{\gamma}(\gamma)\right) u_{\gamma}(\gamma)\right]+\int_{\gamma}^{\alpha_{+}} \frac{d}{d \gamma}\left[W\left(u_{\gamma}(x)\right)-W^{\prime}\left(u_{\gamma}(x)\right) u_{\gamma}(x)\right] d x \\
=\frac{1}{2}( & \left.\frac{1}{\varepsilon_{+}}-\frac{1}{\varepsilon_{-}}\right)\left[\varepsilon_{\gamma} u_{\gamma}^{\prime}(\gamma)\right]^{2} \\
& -\int_{\alpha_{-}}^{\gamma} u_{\gamma}^{\prime}(x)\left[f(\gamma)+\varepsilon_{-} c_{-}^{\prime}(\gamma)\right] d x-\int_{\gamma}^{\alpha_{+}} u_{\gamma}^{\prime}(x)\left[f(\gamma)+\varepsilon_{+} c_{+}^{\prime}(\gamma)\right] d x \\
& -\int_{\gamma}^{\alpha_{+}} u_{\gamma}^{\prime}(x)\left[-W^{\prime}\left(u_{\gamma}(\gamma)\right)+\int_{\gamma}^{x} \frac{d}{d \gamma} W^{\prime}\left(u_{\gamma}(y)\right) d y\right] d x \\
& -\left[W\left(u_{\gamma}(\gamma)\right)-W^{\prime}\left(u_{\gamma}(\gamma)\right) u_{\gamma}(\gamma)\right]+\int_{\gamma}^{\alpha_{+}} \frac{d}{d \gamma}\left[W\left(u_{\gamma}(x)\right)-W^{\prime}\left(u_{\gamma}(x)\right) u_{\gamma}(x)\right] d x \\
\frac{1}{2} & \left(\frac{1}{\varepsilon_{+}}-\frac{1}{\varepsilon_{-}}\right)\left[\varepsilon_{\gamma} u_{\gamma}^{\prime}(\gamma)\right]^{2}-\left[f(\gamma)+\varepsilon_{-} c_{-}^{\prime}(\gamma)\right] \int_{\alpha_{-}}^{\alpha_{+}} u_{\gamma}^{\prime}(x) d x \\
& +W^{\prime}\left(u_{\gamma}(\gamma)\right) \int_{\gamma}^{\alpha_{+}} u_{\gamma}^{\prime}(x) d x-\int_{\gamma}^{\alpha_{+}} u_{\gamma}^{\prime}(x)\left[\int_{\gamma}^{x} \frac{d}{d \gamma} W^{\prime}\left(u_{\gamma}(y)\right) d y\right] d x \\
& -W_{(}\left(u_{\gamma}(\gamma)\right)+W^{\prime}\left(u_{\gamma}(\gamma)\right) u_{\gamma}(\gamma)+\int_{\gamma}^{\alpha_{+}} \frac{d}{d \gamma}\left[W\left(u_{\gamma}(x)\right)-W^{\prime}\left(u_{\gamma}(x)\right) u_{\gamma}(x)\right] d x \\
=\frac{1}{2} & \left(\frac{1}{\varepsilon_{+}}-\frac{1}{\varepsilon_{-}}\right)\left[\varepsilon_{\gamma} u_{\gamma}^{\prime}(\gamma)\right]^{2}+\int_{\gamma}^{\alpha_{+}} u_{\gamma}(x) \frac{d}{d \gamma}\left(W^{\prime}\left(u_{\gamma}(x)\right)\right) d x-W_{\gamma}\left(u_{\gamma}(\gamma)\right) \\
& -\int_{\gamma}^{\alpha_{+}} W^{\prime \prime}\left(u_{\gamma}(x)\right) u_{\gamma}(x) \frac{d}{d \gamma} u_{\gamma}(x) d x \\
\frac{1}{2}( & \left.\frac{1}{\varepsilon_{+}}-\frac{1}{\varepsilon_{-}}\right)\left[\varepsilon_{\gamma} u_{\gamma}^{\prime}(\gamma)\right]^{2}-W_{\gamma}\left(u_{\gamma}(\gamma)\right) .
\end{aligned}
$$

This is (4.2).

\section{Appendix B.}

We calculate each term in (4.3). By (4.6) and the fact that $\psi_{v a c}(x)=Q /\left(\varepsilon_{-}|x|\right)$ in the current setting, we have

$$
\frac{1}{2} Q\left(\psi_{R}-\psi_{v a c}\right)(0)=\frac{Q^{2}}{2 R}\left(\frac{1}{\varepsilon_{+}}-\frac{1}{\varepsilon_{-}}\right)-\frac{\kappa Q^{2}}{2 \varepsilon_{+}(1+\kappa R)} .
$$

It follows from the continuity of $\psi_{R}$ and $\varepsilon_{R} \nabla \psi_{R}$ across the boundary $\Gamma_{R}$ (cf. the third and fourth equations of (4.5)) and (4.6),

$$
-\frac{\varepsilon_{-} R^{2}}{2} \psi_{R}(R) \psi_{R}^{\prime}(R-)=-\frac{\varepsilon_{-} R^{2}}{2} \psi_{R}(R+) \psi_{R}^{\prime}(R-)=\frac{Q^{2}}{2 \varepsilon_{+} R(1+\kappa R)} .
$$


By (4.6) and integration by parts, we have

$$
\begin{aligned}
-\frac{\varepsilon_{+}}{2} \int_{R}^{\infty}\left|\psi_{R}^{\prime}(r)\right|^{2} r^{2} d r & =-\frac{Q^{2}}{2 \varepsilon_{+}(1+\kappa R)^{2}} \int_{R}^{\infty}\left[\frac{d}{d r}\left(\frac{e^{-\kappa(r-R)}}{r}\right)\right]^{2} r^{2} d r \\
& =-\frac{Q^{2}}{2 \varepsilon_{+}(1+\kappa R)^{2}} \int_{R}^{\infty} \frac{(1+\kappa r)^{2}}{r^{2}} e^{-2 \kappa(r-R)} d r \\
& =-\frac{Q^{2}}{2 \varepsilon_{+}(1+\kappa R)^{2}}\left\{\kappa^{2} \int_{R}^{\infty} e^{-2 \kappa(r-R)} d r-\int_{R}^{\infty} \frac{d}{d r}\left[\frac{e^{-2 \kappa(r-R)}}{r}\right] d r\right\} \\
& =-\frac{\kappa Q^{2}}{4 \varepsilon_{+}(1+\kappa R)^{2}}-\frac{Q^{2}}{2 \varepsilon_{+} R(1+\kappa R)^{2}} .
\end{aligned}
$$

The combination of these three equations, (4.3), and (4.6) leads to (4.7).

Taylor expanding the exponential term in the last integral of (4.7) up to $\psi_{R}^{2}$ and using the electrostatic neutrality $\sum_{j=1}^{M} q_{j} c_{j}^{\infty}=0$, we obtain by (4.6) and a simple calculation the approximation

$$
-2 \pi \beta \sum_{j=1}^{\infty} q_{j}^{2} c_{j}^{\infty} \int_{R}^{\infty}\left[\psi_{R}(r)\right]^{2} r^{2} d r=-\frac{\kappa Q^{2}}{4 \varepsilon_{+}(1+\kappa R)^{2}}
$$

Replacing the last integral in (4.7) by this term, we obtain the BDH formula (4.8).

\section{Appendix C.}

The PB equation (4.9) and the boundary condition $\psi_{R}(\infty)=0$ are equivalent to

$$
\left\{\begin{array}{l}
\varepsilon_{-} \nabla^{2} \psi_{R}=-4 \pi Q \delta(x) \quad \text { for }|x|<R \\
\varepsilon_{+} \nabla^{2} \psi_{R}-4 \pi \sum_{j=1}^{M} q_{j} c_{j}^{\infty} e^{-\beta q_{j} \psi_{R}}=0 \quad \text { for }|x|>R \\
\psi_{R}(R-0)=\psi_{R}(R+0) \\
\varepsilon_{-} \psi_{R}^{\prime}(R-0)=\varepsilon_{+} \psi_{R}^{\prime}(R+0) \\
\psi_{R}(\infty)=0
\end{array}\right.
$$

Since $\psi_{R}$ is spherically symmetric, the second equation in (C.1) becomes

$$
\frac{d}{d r}\left(r^{2} \frac{d \psi_{R}}{d r}\right)=-\frac{4 \pi}{\varepsilon_{+}} r^{2} \sum_{j=1}^{M} q_{j} c_{j}^{\infty} e^{-\beta q_{j} \psi_{R}(r)}=: W_{R}(r) .
$$

Solving (C.1), we obtain

$$
\psi_{R}(r)=\left\{\begin{array}{l}
\frac{Q}{\varepsilon_{-} r}+d_{-}(R) \quad \text { for }|x|<R \\
-\int_{r}^{\infty} V_{R}(s) d s-\frac{c_{+}(R)}{r} \quad \text { for }|x|>R,
\end{array}\right.
$$


where

$$
\begin{aligned}
& c_{+}(R)=-\frac{Q}{\varepsilon_{+}}+\int_{R}^{\infty} W_{R}(r) d r \\
& d_{-}(R)=\frac{Q}{R}\left(\frac{1}{\varepsilon_{+}}-\frac{1}{\varepsilon_{-}}\right)-\int_{R}^{\infty} V_{R}(r) d r-\frac{1}{R} \int_{R}^{\infty} W_{R}(r) d r
\end{aligned}
$$

are two constants with respect to $x \in \mathbb{R}^{3}$ but are dependent on $R$, and

$$
\begin{aligned}
& V_{R}(r)=-\frac{1}{r^{2}} \int_{r}^{\infty} W_{R}(s) d s \\
& W_{R}(r)=-\frac{4 \pi}{\varepsilon_{+}} r^{2} \sum_{j=1}^{M} q_{j} c_{j}^{\infty} e^{-\beta q_{j} \psi_{R}(r)} .
\end{aligned}
$$

By (4.3), the continuity of $\psi_{R}$ and $\varepsilon_{R} \nabla \psi_{R}$ across the boundary $\Gamma_{R}$ (cf. the third and fourth equations of (C.1)), and (C.3), we obtain

$$
G[R]=Q d_{-}(R)+\frac{Q^{2}}{2 \varepsilon_{-} R}-\frac{\varepsilon_{+}}{2} \int_{R}^{\infty}\left|\psi_{R}^{\prime}(r)\right|^{2} r^{2} d r-4 \pi \beta^{-1} \sum_{j=1}^{M} c_{j}^{\infty} \int_{R}^{\infty}\left[e^{-\beta q_{j} \psi_{R}(r)}-1\right] r^{2} d r .
$$

Consequently,

$$
\begin{aligned}
G^{\prime}[R] & =Q d_{-}^{\prime}(R)-\frac{Q^{2}}{2 \varepsilon_{-} R^{2}}+\frac{\varepsilon_{+}}{2}\left|\psi_{R}^{\prime}(R+0)\right|^{2} R^{2}-\int_{R}^{\infty} \frac{\varepsilon_{+}}{2} r^{2} \frac{d}{d R}\left(\left|\psi_{R}^{\prime}(r)\right|^{2}\right) d r \\
& +4 \pi \beta^{-1} \sum_{j=1}^{M} c_{j}^{\infty}\left[e^{-\beta q_{j} \psi_{R}(R)}-1\right] R^{2}+4 \pi \sum_{j=1}^{M} q_{j} c_{j}^{\infty} \int_{R}^{\infty} e^{-\beta q_{j} \psi_{R}(r)}\left(\frac{d}{d R} \psi_{R}(r)\right) r^{2} d r .
\end{aligned}
$$

It follows from (C.1) and (C.3) that

$$
\frac{\varepsilon_{+}}{2}\left|\psi_{R}^{\prime}(R+0)\right|^{2} R^{2}=\frac{R^{2}}{2 \varepsilon_{+}}\left|\varepsilon_{+} \psi_{R}^{\prime}(R+0)\right|^{2}=\frac{R^{2}}{2 \varepsilon_{+}}\left|\varepsilon_{-} \psi_{R}^{\prime}(R-0)\right|^{2}=\frac{Q^{2}}{2 \varepsilon_{+} R^{2}} .
$$

By integration by parts, (C.2), (C.5), (C.1), and (C.3),

$$
\begin{aligned}
-\int_{R}^{\infty} & \frac{\varepsilon_{+}}{2} r^{2} \frac{d}{d R}\left(\left|\psi_{R}^{\prime}(r)\right|^{2}\right) d r \\
= & -\int_{R}^{\infty} \varepsilon_{+} r^{2} \psi_{R}^{\prime}(r)\left(\frac{d}{d R} \psi_{R}^{\prime}(r)\right) d r \\
& =-\int_{R}^{\infty} \varepsilon_{+} r^{2} \psi_{R}^{\prime}(r) \frac{d}{d r}\left(\frac{d}{d R} \psi_{R}(r)\right) d r \\
& =\left.\left[-\varepsilon_{+} r^{2} \psi_{R}^{\prime}(r)\left(\frac{d}{d R} \psi_{R}(r)\right)\right]\right|_{r=R+} ^{r=\infty}+\int_{R}^{\infty} \varepsilon_{+} \frac{d}{d r}\left(r^{2} \psi_{R}^{\prime}(r)\right)\left(\frac{d}{d R} \psi_{R}(r)\right) d r
\end{aligned}
$$




$$
\begin{aligned}
& =\left.\varepsilon_{+} R^{2} \psi_{R}^{\prime}(R+)\left(\frac{d}{d R} \psi_{R}(r)\right)\right|_{r=R+}+\int_{R}^{\infty} \varepsilon_{+} W_{R}(r)\left(\frac{d}{d R} \psi_{R}(r)\right) d r \\
& =-\left.Q\left(\frac{d}{d R} \psi_{R}(r)\right)\right|_{r=R+}-4 \pi \sum_{j=1}^{M} q_{j} c_{j}^{\infty} \int_{R}^{\infty} r^{2} e^{-\beta q_{j} \psi_{R}(r)}\left(\frac{d}{d R} \psi_{R}(r)\right) d r .
\end{aligned}
$$

Combining the above three equations, we have

$$
\begin{gathered}
G^{\prime}[R]=\frac{Q^{2}}{2 R^{2}}\left(\frac{1}{\varepsilon_{+}}-\frac{1}{\varepsilon_{-}}\right)+4 \pi R^{2} \beta^{-1} \sum_{j=1}^{M} c_{j}^{\infty}\left[e^{-\beta q_{j} \psi_{R}(R)}-1\right] \\
+Q d_{-}^{\prime}(R)-\left.Q\left(\frac{d}{d R} \psi_{R}(r)\right)\right|_{r=R+} .
\end{gathered}
$$

Notice that

$$
\frac{d}{d R}\left(\psi_{R}(R+)\right)=\left.\left(\frac{d}{d R} \psi_{R}\right)(r)\right|_{r=R+}+\psi_{R}^{\prime}(R+) .
$$

This, together with the continuity of $\psi_{R}$ and $\varepsilon_{R} \psi_{R}^{\prime}$ at $r=R$ and (C.3), implies that

$$
\begin{aligned}
-\left.Q\left(\frac{d}{d R} \psi_{R}(r)\right)\right|_{r=R+} & =-Q\left[\frac{d}{d R}\left(\psi_{R}(R+)\right)-\psi_{R}^{\prime}(R+)\right] \\
& =-Q \frac{d}{d R}\left(\psi_{R}(R-)\right)+\frac{Q \varepsilon_{+} \psi_{R}^{\prime}(R+)}{\varepsilon_{+}} \\
& =-Q \frac{d}{d R}\left[\frac{Q}{\varepsilon_{-} R}+d_{-}(R)\right]+\frac{Q \varepsilon_{-} \psi_{R}^{\prime}(R-)}{\varepsilon_{+}} \\
& =\frac{Q^{2}}{\varepsilon_{-} R^{2}}-Q d_{-}^{\prime}(R)-\frac{Q^{2}}{\varepsilon_{+} R^{2}}
\end{aligned}
$$

The insertion of this into (C.6) leads to (4.10).

Acknowledgments. This work is partially supported by the Deutsche Forschungsgemeinschaft (DFG) within the Emmy-Noether Programme (J. D.), by the US National Science Foundation (NSF) through grant DMS-0451466 (B. L.), and by the US Department of Energy through grant DE-FG02-05ER25707 (B. L.). Work in the McCammon group is supported in part by NSF, NIH, HHMI, CTBP, NBCR, and Accelrys.

\section{References}

[1] Chu, B. Molecular Forces, Based on the Lecture of Peter J. W. Debye; Interscience, John Wiley \& Sons, 1967. 
[2] Israelachvili, J. Intermolecular and Surface Forces; Acdemic Press, 2nd ed., 1991.

[3] Reiner, E. S.; Radke, C. J. J. Chem. Soc. Faraday Trans. 1990, 86, 3901-3912.

[4] Sharp, K. A.; Honig, B. J. Phys. Chem. 1990, 94, 7684-7692.

[5] Borukhov, I.; Andelman, D.; Orland, H. Phys. Rev. Lett. 1997, 79, 435-438.

[6] Fogolari, F.; Briggs, J. M. Chem. Phys. Lett. 1997, 281, 135-139.

[7] Fogolari, F.; Zuccato, P.; Esposito, G.; Viglino, P. Biophys. Journal 1999, 76, 1-16.

[8] Gilson, M. K.; Davis, M. E.; Luty, B. A.; McCammon, J. A. J. Phys. Chem 1993, 97, 3591-3600.

[9] Chou, T. IPAM Lecture Notes 2002.

[10] Allen, R.; Hansen, J.-P.; Melchionna, S. Phys. Chem. Chem. Phys. 2001, 3, 41774186.

[11] Kralj-Iglic, V.; Iglic, A. J. Phys. II (France) 1996, 6, 477-491.

[12] Roux, B.; Simonson, T. Biophys. Chem. 1999, 78, 1-20.

[13] Feig, M.; III, C. L. B. Current Opinion in Structure Biology 2004, 14, 217-224.

[14] Richards, F. M. Annu. Rev. Biophys. Bioeng. 1977, 6, 151-176.

[15] Davis, M. E.; McCammon, J. A. Chem. Rev. 1990, 90, 509-521.

[16] Fixman, M. J. Chem. Phys. 1979, 70, 4995-5005.

[17] Still, W. C.; Tempczyk, A.; Hawley, R. C.; Hendrickson, T. J. Amer. Chem. Soc. 1990, 112, 6127-6129.

[18] Bashford, D.; Case, D. A. Ann. Rev. Phys. Chem 2000, 51, 129-152.

[19] Dzubiella, J.; Swanson, J. M. J.; McCammon, J. A. Phys. Rev. Lett. 2006, 96, 087802.

[20] Dzubiella, J.; Swanson, J. M. J.; McCammon, J. A. J. Chem. Phys. 2006, 124, 084905.

[21] Dzubiella, J.; Allen, R. J.; Hansen, J. P. J. Chem. Phys. 2004, 120, 5001-5004.

[22] Smeets, R. M. M.; Keyser, U. F.; Wu, M. Y.; Dekker, N. H.; Dekker, C. Phys. Rev. Lett. 2006, 97, 088101.

[23] Born, M. Z. Phys. 1920, 1, 45-48. 